\title{
Surfactant-Facilitated Crystallization of Dihydrate Carbamazepine during Dissolution of Anhydrous Polymorph
}

\author{
N. RODRÍGUEZ-HORNEDO, D. MURPHY \\ Department of Pharmaceutical Sciences, College of Pharmacy, University of Michigan, 428 Church Street, \\ Ann Arbor, Michigan 48109-1065
}

Received 26 November 2002; revised 21 February 2003; accepted 22 May 2003

\begin{abstract}
The influence of two structurally different anionic surfactants on the anhydrous-to-dihydrate transformation of carbamazepine (CBZ) was investigated. The surfactants studied were sodium lauryl sulfate (SLS), a surfactant commonly used in compendial dissolution methods, and sodium taurocholate (STC), an important surfactant in the solubilization and absorption of drugs and lipids in the gastrointestinal tract. Results show that both surfactants promoted the crystallization of CBZ dihydrate $[\mathrm{CBZ}(\mathrm{D})]$ during dissolution of the anhydrous monoclinic polymorph [CBZ(A)]). Examination of crystal surfaces showed that SLS facilitated the surface-mediated nucleation of $\mathrm{CBZ}(\mathrm{D})$ on $\mathrm{CBZ}(\mathrm{A})$ crystals at surfactant concentrations below the critical micelle concentration (cmc). Solubilization of a dye and related color changes provided visual evidence for adsorbed SLS assemblies on CBZ(A) crystal faces below the cmc. Above the cmc, both surfactants promoted the transformation by increasing the bulk nucleation of CBZ(D). STC changed the crystal morphology of CBZ(D) from acicular to prismatic, depending on STC concentration. Such morphology changes originate from interactions between STC and molecular structures of CBZ(D) crystal faces that interfere with the formation of a hydrogen-bonded chain of water molecules and carboxamide dimers.

(C) 2004 Wiley-Liss, Inc. and the American Pharmacists Association J Pharm Sci 93:449-460, 2004

Keywords: co-crystals; clathrates; crystal engineering; crystal structure; crystallization; hydrates/solvates; polymorphism
\end{abstract}

\section{INTRODUCTION}

The range of solid state forms that a drug substance exhibits (i.e., amorphous, polymorphs, solvates, or co-crystals) is a key issue in the development of a pharmaceutical product because the form can affect bioavailability and stability in significant ways. $^{1,2}$ In fact, transformations between solid state forms can compromise dissolution behavior ${ }^{3-5}$ and the physical and chemical

D. Murphy's present address is Bristol-Myers Squibb Pharmaceutical Research Institute, Princeton, NJ

Correspondence to: N. Rodríguez-Hornedo (Telephone: 734763-0101; Fax: 734-615-6162; E-mail: nrh@umich.edu)

Journal of Pharmaceutical Sciences, Vol. 93, 449-460 (2004)

(c) 2004 Wiley-Liss, Inc. and the American Pharmacists Association integrity of a drug product. ${ }^{6,7}$ During dissolution tests, for instance, solution-mediated transformations are important because the rate of the transformation determines the concentration of drug in solution, with faster conversion rates leading to lower levels of drug dissolved. ${ }^{8,9}$

Solution-mediated transformations involve three main steps: dissolution of a metastable solid phase, nucleation, and growth of a stable solid phase. Studies in the pharmaceutical literature have focused on the dissolution process and the effect of surfactants, ${ }^{10-12}$ with little attention given to how surfactants affect the crystallization steps involved in solution-mediated transformations. It would seem that surfactants, given their interfacial properties and their ability to form a variety of supramolecular structures (micelles, 
hemimicelles, etc.) in aqueous solution, could have a profound effect on crystallization in addition to their effects on dissolution. Surfactants have been shown to promote or inhibit crystallization steps (nucleation, crystal growth) in organic and inorganic crystals ${ }^{13-18}$ and direct the crystallization of solid phases that otherwise are not formed. ${ }^{19,20}$

In this paper, we examine the role of physiologically relevant surfactants and conventional surfactants on the dissolution behavior of metastable solid phases. Previous work has shown that the monoclinic polymorph of carbamazepine anhydrous [CBZ(A)] converts to the dihydrate form, CBZ(D), in aqueous solution via a solutionmediated mechanism. ${ }^{21-23}$ Carbamazepine (CBZ) is an anticonvulsant with a narrow range of therapeutic efficacy, and commercial tablets of CBZ are formulated with the anhydrous monoclinic form, CBZ(A). The potential for a solution-mediated transformation to the dihydrate crystal form, CBZ(D), lies in the fact that CBZ(A) has a solubility at room temperature that is at least three times greater than that of CBZ(D) in water. ${ }^{23}$ As with other poorly water soluble drugs, the compendial dissolution method for CBZ tablets requires the use of $1 \%$ sodium lauryl sulfate (SLS). ${ }^{24}$ Surfactants have been shown to promote or inhibit the growth of CBZ(D) in suspensions of CBZ(A) depending on their solubilizing capacity. ${ }^{16,25,26}$ Polysorbate 80 and poloxamer 184 slowed CBZ(D) growth, whereas SLS and benzalkonium chloride accelerated growth. Because surfactants can affect both dissolution and crystallization processes, it is important to know how synthetic and biological surfactants affect the dissolution performance of a drug that undergoes a solution-mediated transformation.

The aim of the present study was to investigate the influence of two structurally different anionic surfactants on the anhydrous monoclinic-to-dihydrate CBZ transformation in aqueous solutions. The surfactants studied were SLS, a conventional surfactant, and sodium taurocholate (STC), an important surfactant in the solubilization and absorption of drugs and lipids in the gastrointestinal tract. These surfactants have different physicochemical properties ${ }^{12,27}$ because of their molecular structures and self-organization behavior. ${ }^{28,29}$ SLS has a flexible alkyl chain that exhibits axial polarity (hydrophilic head group and hydrophobic tail), whereas STC is a trihydroxy bile salt, with a rigid steroid ring system that exhibits facial polarity (hydrophilic and hydrophobic planes). Molecular structures of carbamazepine and surfactants are shown in Figure 1. Experiments were carried out to monitor the drug concentration and solid phase composition during the transformation, and to examine crystal surfaces and morphologies of dissolving $\mathrm{CBZ}(\mathrm{A})$ and crystallizing CBZ(D). The influence of surfactants on the phase transformation and dissolution behavior is explained on the basis of molecular structures of crystal surfaces and molecular and supramolecular structures of surfactants.

\section{EXPERIMENTAL}

\section{Materials}

Anhydrous monoclinic carbamazepine [CBZ(A), lot \# 28F0109) was purchased from Sigma Chemical Company (St. Louis, MO) and used as received. CBZ(A) was also recrystallized from methanol (Fisher Scientific, HPLC grade) by cooling a warm solution of CBZ $(0.14 \mathrm{~g} / \mathrm{mL})$ to $10^{\circ} \mathrm{C}$ and harvesting the solid phase after $20 \mathrm{~min}$ at $10^{\circ} \mathrm{C}$. Large single crystals of CBZ(A) were grown by slow evaporation at room temperature of solutions containing CBZ in methanol $(0.02-0.05 \mathrm{~g} / \mathrm{mL})$. Carbamazepine dihydrate [CBZ(D)] was prepared by crystallization from aqueous solutions supersaturated with respect to the dihydrate form and

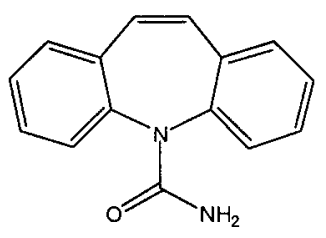

(a)

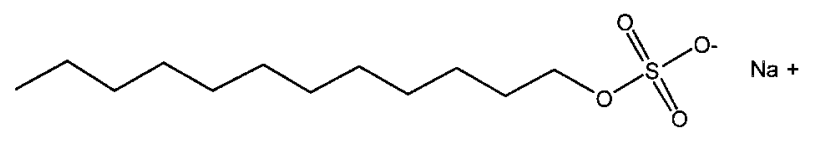

(b)

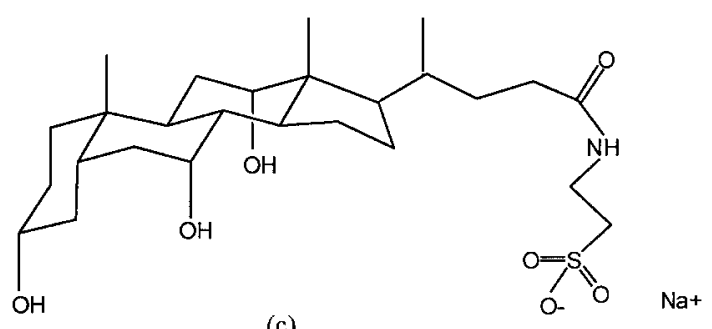

(c)

Figure 1. Chemical structures of (a) carbamazepine, (b) sodium lauryl sulfate, and (c) sodium taurocholate. 
used for solubility and dissolution studies. Supersaturation was created by dissolving $\mathrm{CBZ}(\mathrm{A})$ at $50-65^{\circ} \mathrm{C}$ with constant stirring, filtering the solution to remove any extraneous particulates, and cooling the filtrate to $5^{\circ} \mathrm{C}$.

Sodium taurocholate (STC, 98\% purity) and sodium lauryl sulfate (SLS, 99\% purity) were purchased from Sigma Chemical Company (St. Louis, MO) and used as received. The surfactant concentrations studied were above and below the critical micellar concentration (cmc) for each surfactant. The highest surfactant concentration studied was $17.3 \mathrm{mM}$ because of the fast conversion rates of CBZ(A) to CBZ(D) at concentrations of SLS above this value. It is worth noting that this value corresponds to half of the concentration of SLS required in the USP dissolution method for CBZ tablets. ${ }^{24}$ The water used in this study was filtered through a double-deionized purification system (Milli Q Plus Water System from Millipore Company, Bedford, MA). Pinacyanol chloride (PC), used in adsolubilization studies, was purchased from Sigma Chemical Company (St. Louis, MO) and used as received.

\section{Solubility of Anhydrous and Dihydrate CBZ}

The solubility of both $\mathrm{CBZ}(\mathrm{D})$ and $\mathrm{CBZ}(\mathrm{A})$ was determined by the equilibrium and dissolution methods as described in a previous publication. ${ }^{23}$ Solubilities reported in the present work were measured in aqueous solutions at surfactant concentrations in the range $2.0-17.3 \mathrm{mM}$ at $25^{\circ} \mathrm{C}$.

\section{Transformation of Anhydrous to Dihydrate CBZ}

The transformation from CBZ(A) to CBZ (D) was studied from suspensions of CBZ(A) in solutions initially supersaturated with respect to the dihydrate phase at $25^{\circ} \mathrm{C}$ by the methods described in detail previously. ${ }^{23}$ The solution-mediated transformation of CBZ(A) to CBZ(D) was characterized by monitoring the following: CBZ concentration in solution, solid phase composition, and crystal morphology during the transformation. The studies reported here were carried out at a supersaturation of 0.15 or 1.15 times the solubility of the dihydrate form in all the surfactant solutions. Supersaturation is defined as $\sigma_{\mathrm{D}}=\left(C / S_{\mathrm{D}}\right)-1$, where $C$ is the CBZ concentration in solution and $S_{\mathrm{D}}$ the solubility of CBZ(D).

Solutions were pre-supersaturated at $25^{\circ} \mathrm{C}$ by dissolving $\mathrm{CBZ}(\mathrm{A})$ at $60^{\circ} \mathrm{C}$ in surfactant solutions, filtering through a $0.22-\mu \mathrm{m}$ filter, and quickly cooling to $25^{\circ} \mathrm{C}$ in ice. Five hundred milligrams of CBZ(A) and $250 \mathrm{~mL}$ of the solution supersaturated with respect to $\mathrm{CBZ}(\mathrm{D})$ were added to a jacketed beaker, with temperature controlled at $25 \pm 1^{\circ} \mathrm{C}$ (Neslab RTE 210, Portsmouth, NH). The specific surface area of CBZ(A) was $0.07 \mathrm{~m}^{2} / \mathrm{g}$ as measured by the Brunauer-Emmett-Teller method (Quantasorb Surface Area Analyzer, Quantachrome Company, Boynton Beach, FL). The suspension was stirred at $400 \mathrm{rpm}$. Aliquots of the suspension were withdrawn at various time intervals with a plastic syringe and filtered through a $0.45-\mu \mathrm{m}$ Millipore filter in a $13-\mathrm{mm}$ Swinex disc filter holder. The filtrate was appropriately diluted, and the concentration of CBZ in solution was measured by ultraviolet (UV) spectrophotometry at $284.5 \mathrm{~nm}$ with a Perkin-Elmer Lambda $7 \mathrm{UV}-$ visible (UV-vis) spectrophotometer (Shelton, CT).

The methods for analyzing the solid phase during the transformation have been described previously, ${ }^{23}$ including X-ray powder diffraction (XRPD), differential scanning calorimetry (DSC), and weight loss on drying. At periodic intervals during the transformation of $\mathrm{CBZ}(\mathrm{A})$ to $\mathrm{CBZ}(\mathrm{D})$, $5-10 \mathrm{~mL}$ of the suspension were filtered under reduced pressure $(25 \mathrm{mmHg}$ ) for $40 \mathrm{~min}$ to remove physically bound water. ${ }^{22}$ The weight fraction of CBZ(A) and CBZ(D) was quantified by comparing the percent weight loss of dried samples to a standard curve based on physical mixtures of known composition of CBZ(A) and CBZ(D). An ultramicrobalance (Mettler UM-3) was used to weigh the solid phase $(3-10 \mathrm{mg} \pm 1 \mu \mathrm{g})$.

\section{Crystal Morphology of CBZ(D)}

To identify the morphologically important faces of $\mathrm{CBZ}(\mathrm{D})$, single crystals were grown at $25^{\circ} \mathrm{C}$, in the presence of 2.0-9.0 mM STC and at a constant supersaturation of 1.5 , in a flow cell apparatus previously described by our group. ${ }^{30,31}$ The morphology of crystals in the glass flow cell $(0.25 \times$ $0.50 \times 3.0 \mathrm{~cm})$ was monitored with an inverted microscope (Nikon, Diaphot-TMD, Nikon Inc., Melville, NY) using a $40 \times$ long working distance objective.

The Miller indices of the CBZ(D) crystal faces were calculated from the interfacial angles that were measured with a two-circle reflecting goniometer. The Miller index of each morphologically important crystal face was calculated with the equation that relates the interplanar angles to the unit cell lattice parameters reported for CBZ(D) ${ }^{32}$ The crystallographic axes were verified with a 
precession camera and by comparing the measured interlattice spacings from the zero level precession photographs to the reported lattice parameters.

\section{Microscopy}

CBZ solid was examined during the transformation while suspended in the transformation medium with an inverted light microscope (Nikon, Diaphot-TMD, Nikon Inc., Melville, NY), with $10 \times$ and $40 \times$ Nomarski objectives. Solid phases were also examined, by scanning electron microscopy (SEM; Hitachi S-3200N, Tokyo, Japan). Samples were prepared by transferring the CBZ solid to a strip of double-sided carbon tape (Ted Pella, Inc., Redding, CA) attached to a standard SEM mounting stub. The samples were subsequently coated with gold for 200 s (Desk II, Denton Vacuum, Moorestown, NJ) to prevent charging at the surface. The microscope was operated with $15 \mathrm{kV}$ beam current.

\section{X-ray Powder Diffraction}

The XRPD patterns were recorded using a Scintag X-ray diffractometer (Franklin, MA) using $\mathrm{CuK} \alpha$ radiation $(\lambda=1.5418 \AA)$, tube voltage of $35-45 \mathrm{kV}$, tube current of $20-40 \mathrm{~mA}, 2 \theta$ of $2-50^{\circ}$, scan rate of $5^{\circ} / \mathrm{min}$, and step size of $0.020^{\circ}$.

\section{Differential Scanning Calorimetry}

The thermal properties of CBZ were determined by DSC with a Perkin-Elmer DSC-7 (Shelton, CT). Samples were weighed into aluminum pans that were then sealed with a crimper. The thermal behavior was studied under a dry nitrogen purge $(20 \mathrm{~mL} / \mathrm{min})$ at a heating rate of $10^{\circ} \mathrm{C} /$ $\min$.

\section{Adsolubilization}

The method described by Nun et al. ${ }^{33}$ for visual evidence of solubilization of a dye on an alumina surface as a result of surfactant aggregation or surface micelle formation was used to test for the presence of adsorbed surfactant layers that can adsolubilize solute molecules. PC was added to suspensions of CBZ(A) containing $4.3 \mathrm{mM}$ SLS to achieve a dye concentration of $1 \times 10^{-5} \mathrm{M}$. The systems were initially undersaturated with respect to both anhydrous and dihydrate CBZ to retard the anhydrous-to-dihydrate CBZ trans- formation. Suspensions of CBZ(A) containing PC in the absence of SLS served as a control. Suspensions were placed in scintillation vials and shaken (Speci-Mix mixer, Thermolyne Corp., Dubuque, IA) at room temperature $\left(\sim 22^{\circ} \mathrm{C}\right)$. The solution and solid surfaces were visually examined for color changes. In the presence of SLS, a reddish dye salt-SLS complex forms at concentrations below the cmc, and the solution shows a red color. ${ }^{34}$ When the dye-SLS salt is solubilized and incorporated within surfactant micelles the solution turns blue similar to when PC is dissolved in organic solvents. Even though this method is not reliable for cmc measurement because of the formation of mixed micelles with SLS, it indicates the solubilization of the dye in the hydrocarbon phase of the micelles. This model has been confirmed by Sato et al. with fluorescence decay measurements. ${ }^{35}$

\section{Surface Tension Measurements}

The cmc of SLS was determined in aqueous solutions in the absence and presence of carbamazepine $(0.16 \mathrm{mg} / \mathrm{mL})$ from the surface tension dependence on surfactant concentration. ${ }^{11}$ The surface tension of solutions was measured at $25^{\circ} \mathrm{C}$ by the Wilhelmy Plate method with a platinum plate $^{36}$ (Rosano surface tensiometer, Laboratory Products, Inc., Boston, MA). The instrument was calibrated with known weights, and determination of surface tension was verified by comparison of measured and reported values for water. The cmc was evaluated from the break in the curve of surface tension versus log of surfactant concentration.

\section{RESULTS}

\section{Solubilization by Surfactants}

The solubility of CBZ increases to different degrees in both SLS and STC aqueous solutions. Solubilization curves show that the solubility of $\mathrm{CBZ}(\mathrm{D})$ is equal to its aqueous solubility at surfactant concentrations below the $\mathrm{cmc}$ and increases linearly above the cmc (Figure 2). The solubility of CBZ(D) increased by as much as eight-fold at an SLS concentration of $17.3 \mathrm{mM}$. This SLS concentration corresponds to half of the concentration of SLS required in the dissolution media according to the USP monograph for CBZ tablets. ${ }^{24} \mathrm{CBZ}$ in solution $(0.16 \mathrm{mg} / \mathrm{mL})$ decreased the cmc of SLS 


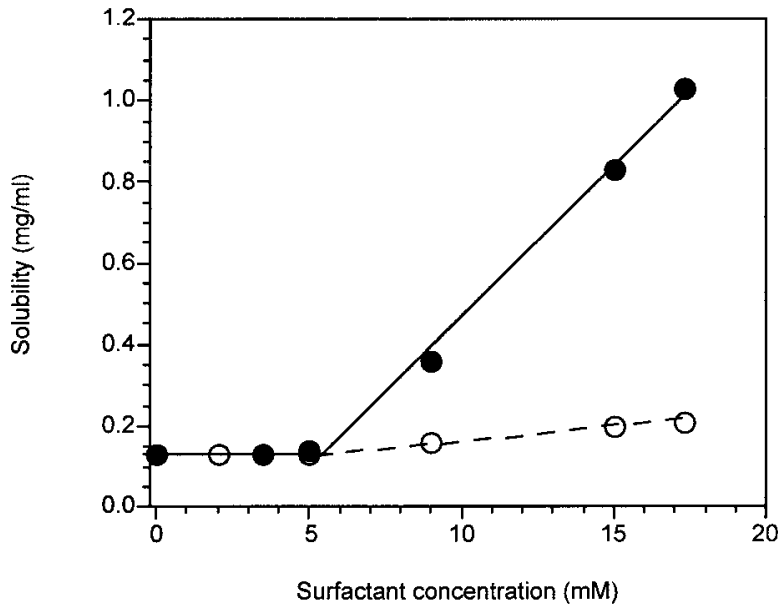

Figure 2. Solubility of $\mathrm{CBZ}(\mathrm{D})$ as a function of surfactant concentration. Key: (O)SLS, (O)STC.

from 6.7 to $5.3 \mathrm{mM}$ at $25^{\circ} \mathrm{C}$. The $\mathrm{cmc}$ in the absence of CBZ is lower than the reported value of $8.3 \mathrm{mM}^{37}$ because of the presence of surface-active impurities and is in agreement with the value reported by Crison et al. ${ }^{11}$

The solubility of CBZ(D) increased 1.7 times in the presence of $17.3 \mathrm{mM}$ STC (Table 1). Solubilization of CBZ was not observed at STC concentrations at or below $5.0 \mathrm{mM}$. The cmc of STC is poorly defined because of the progressive self-association over a range of concentrations, ${ }^{28,38}$ and cmc values in the range $2.5-12.0 \mathrm{mM}$ in water at $25^{\circ} \mathrm{C}$, have been reported. ${ }^{27}$

The molar solubilization capacity, $k$, represents the moles of drug solubilized per mole of surfactant
( $k=S_{\mathrm{m}} / C_{\mathrm{mic}}{ }^{39}$ ) and was evaluated for CBZ(D) according to the following equation:

$$
S_{\mathrm{tot}}=S_{\mathrm{w}}+S_{\mathrm{m}}=S_{\mathrm{w}}+k\left(C_{\mathrm{mic}}\right)
$$

where $C_{\text {mic }}$ is the molar concentration of micellar surfactant, $S_{\mathrm{m}}$ is the molar solubility of the solute in the micellar phase, $S_{\mathrm{w}}$ is the solubility of the solute in water, and $S_{\text {tot }}$ is the total solubility. The molar solubilization capacity for CBZ(D) in SLS is 10 times greater than that in STC (i.e., 0.33 and 0.03 , respectively). The micellar partition coefficient, $K_{\mathrm{m}}\left(=S_{\mathrm{m}} / S_{\mathrm{w}}\right)$, is related to the solubility of the solute in water and was used to estimate the solubilization of CBZ(A) at surfactant concentrations for which the experimental solubility was not measured (Table 1). There is good agreement between the estimated and measured solubility values. The lower values for the experimentally determined solubilities are a result of faster transformation rates to the dihydrate form with increasing surfactant concentration, as discussed later. The solubility of CBZ(A) is about three-fold higher than the solubility of $\mathrm{CBZ}(\mathrm{D})$ at $25^{\circ} \mathrm{C}$, in the presence and absence of surfactants.

\section{Effect of SLS on the Transformation Kinetics}

SLS increased the anhydrous-to-dihydrate CBZ transformation rate at concentrations of $\geq 4.3 \mathrm{mM}$ (Figure 3). At lower SLS concentrations (3.5 mM), dissolution of the anhydrous phase maintained a supersaturated state with respect to the dihydrate form, with undetectable formation of

Table 1. Solubility of Anhydrous and Dihydrate $\mathrm{CBZ}$ at $25^{\circ} \mathrm{C}$ as a Function of Surfactant Concentration

\begin{tabular}{cccc}
\hline & Surfactant $(\mathrm{mM})$ & $\mathrm{CBZ}(\mathrm{A})^{a}(\mathrm{mg} / \mathrm{mL})$ & $\mathrm{CBZ}(\mathrm{D})(\mathrm{mg} / \mathrm{mL})$ \\
\hline SLS & 0 & $0.38 \pm 0.03^{b}[0.38]^{c}$ & $0.125 \pm 0.002$ \\
& 3.5 & $0.38 \pm 0.00[0.38]$ & $0.127 \pm 0.001$ \\
& 5.0 & {$[0.41]$} & $0.135 \pm 0.001$ \\
& 9.0 & {$[1.05]$} & $0.361 \pm 0.001$ \\
& 15.0 & $2.60 \pm 0.26[3.01]$ & $0.828 \pm 0.008$ \\
STC & 17.3 & $0.38 \pm 0.03[0.38]$ & $1.030 \pm 0.003$ \\
& 0 & $0.36 \pm 0.04[0.38]$ & $0.125 \pm 0.002$ \\
& 2.0 & {$[0.38]$} & $0.127 \pm 0.001$ \\
& 5.0 & $0.40 \pm 0.01[0.47]$ & $0.126 \pm 0.001$ \\
& 9.0 & {$[0.58]$} & $0.155 \pm 0.001$ \\
& 15.0 & {$[0.61]$} & $0.196 \pm 0.001$ \\
& 17.3 & &
\end{tabular}

\footnotetext{
${ }^{a}$ Solubility calculated from the intrinsic dissolution rate method.

${ }^{b}$ Mean \pm standard error $(n=3)$.

${ }^{c}$ In brackets, solubility estimated according to the following equation: $\left(S_{\text {tot }} / S_{\mathrm{w}}\right)=1+K_{\mathrm{m}}$.
} 


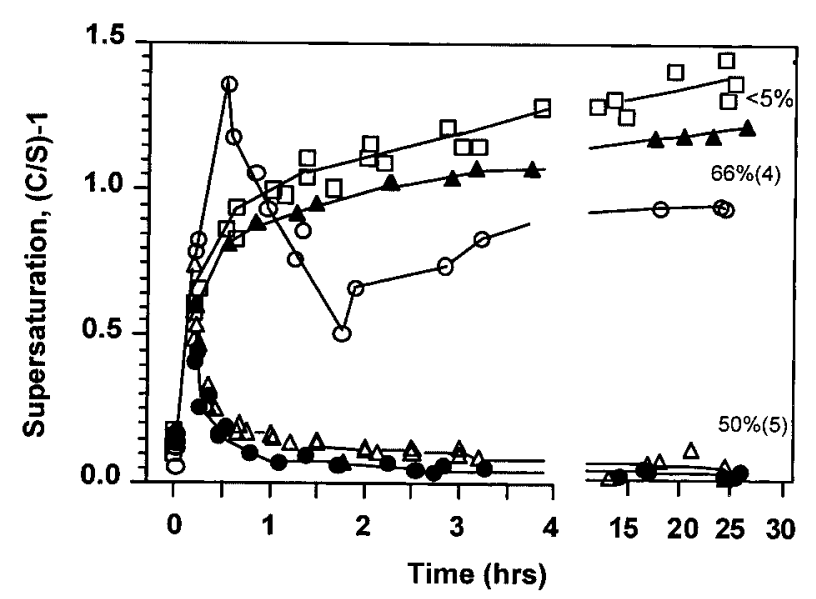

Figure 3. Effect of SLS concentration on the supersaturation profile during the transformation of anhydrous to dihydrate carbamazepine. Initial $(C / S)-1=$ 0.15 at $25^{\circ} \mathrm{C}$. Key to SLS concentration $(\mathrm{mM})$ : (A) 0.0; ( $\square$ ) 3.5; (○) 4.3; $(\triangle)$ 9.0; (○) 17.3. The solid phase composition, percent (w/w) of CBZ(D) in the solid phase with standard deviation in parentheses, is noted for each concentration.

dihydrate in $24 \mathrm{~h}$. This behavior was observed at initial supersaturations as high as 1.31 in $3.5 \mathrm{mM}$ SLS. At $4.3 \mathrm{mM}$ and above, during the initial dissolution stage, CBZ concentrations in solution reached a maximum supersaturation followed by a decrease to a plateau supersaturation as a result of the relative rates of dissolution of CBZ(A) and crystallization of CBZ(D). Both the maximum and plateau concentrations decreased with increasing SLS concentration. This result suggests that SLS decreased the threshold concentration for nucleation and increased the crystallization rate of $\mathrm{CBZ}(\mathrm{D})$ at a faster rate than the dissolution of CBZ(A). Microscopic examination of the solid phase showed needles of CBZ(D) growing on the surface of the dissolving CBZ(A), which is evidence of a heterogeneous nucleation mechanism (Figure 4). Solid-phase analysis showed as much as $50 \% \mathrm{CBZ}(\mathrm{A})$ remaining undissolved by $24 \mathrm{~h}$ for the 9.0 and $17.3 \mathrm{mM}$ SLS, despite steady-state concentrations close to the equilibrium solubility of CBZ(D). This result is because of the extensive surface nucleation of CBZ(D) that decreases the surface of CDZ(A) in contact with the solution phase.

Monitoring $\mathrm{CBZ}(\mathrm{A})$ single crystals during the transformation showed that surface-mediated nucleation of $\mathrm{CBZ}(\mathrm{D})$ on $\mathrm{CBZ}(\mathrm{A})$ increases with SLS concentration and that it is not specific to a given CBZ(A) crystal face. CBZ(A) single crystals grown by slow evaporation from 2 and $5 \% \mathrm{CBZ}$ in

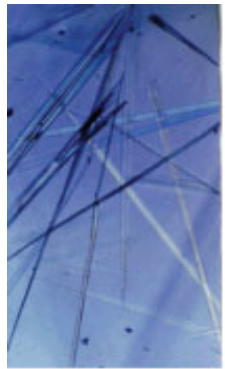

(a)

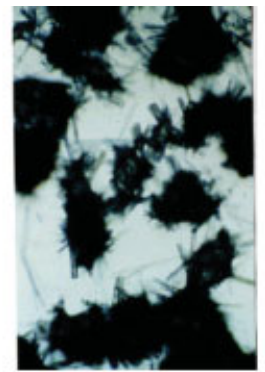

(b)

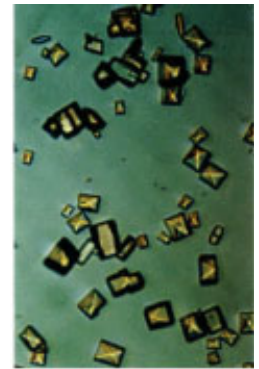

(c)
Figure 4. Photomicrographs of CBZ(D) crystals grown during transformation experiments, at initial supersaturation of 0.15 and $25^{\circ} \mathrm{C}$ : (a) absence of surfactants, (b) $17.3 \mathrm{mM}$ SLS, and (c) $17.3 \mathrm{mM}$ STC. Scale Bar: (一) $200 \mu \mathrm{m}$.

methanol solutions exhibit well developed $\{001\}$, $\{111\}$, and $\{101\}$ faces (Figure 5) and have a morphology that is similar to that of commercially available CBZ(A) crystals. Needles of CBZ(D) grew at random orientations on all faces of single crystals of CBZ(A) within $20 \mathrm{~min}$ in $17.3 \mathrm{mM}$ SLS at initial supersaturation of 0.15 , whereas surface nucleation was not observed for $24 \mathrm{~h}$ in the absence of surfactant (Figure 5).

To understand the heterogeneous nucleation of CBZ(D) and the association of SLS on the CBZ(A) surface, studies were done with a dye (PC) that changes color as a result of micellar solubilization. $\mathrm{PC}$, a dye of the cyanine class, has been used to provide visual evidence for the presence of adsorbed surfactant layers that can adsolubilize solute

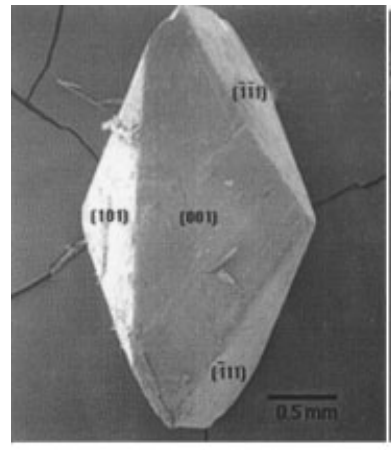

(a)

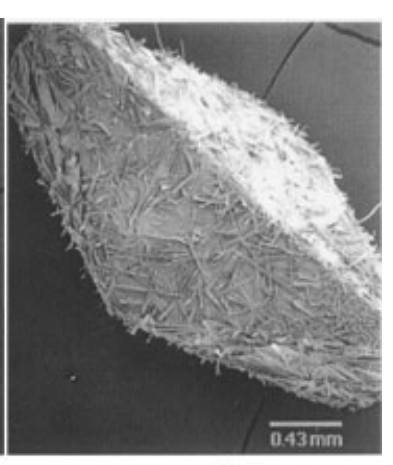

(b)
Figure 5. Photomicrographs of single crystals of CBZ(A) during the transformation to CBZ(D) in the presence of SLS. The characteristic morphology of crystals grown by slow evaporation of 2 and $5 \% \mathrm{w} / \mathrm{w}$ $\mathrm{CBZ}$ in methanol at $25^{\circ} \mathrm{C}$ is shown: (a) crystals placed in aqueous solutions without surfactant at $(C / S)-1=0.15$ for $24 \mathrm{~h}$; and (b) crystals placed in a solution of $17.3 \mathrm{mM}$ SLS at $(C / S)-1=0.15$ for $20 \mathrm{~min}$. 
molecules. ${ }^{33}$ At SLS concentration of $4.3 \mathrm{mM}$, a value below the cmc, the color of CBZ(A) crystal surface became blue, which is characteristic of PC solubilized in micelle. In contrast, the supernatant solution remained red, which is characteristic of $\mathrm{PC}$ in a hydrophilic environment. When suspended in aqueous solutions containing $\mathrm{PC}$ in the absence of SLS, the color of the CBZ(A) crystal surface remained white. These observations suggest that $\mathrm{PC}$ is solubilized into a micelle-like environment on the surface of the CBZ(A) crystals as a result of two-dimensional assemblies of SLS.

\section{Effect of STC on the Transformation Kinetics}

STC increased the anhydrous-to-dihydrate CBZ transformation (Figure 6) at surfactant concentration of $17.3 \mathrm{mM}$, and $78 \%$ of the solid phase consisted of the dihydrate form by $24 \mathrm{~h}$. At STC concentrations of $\leq 9 \mathrm{mM}$, formation of CBZ(D) was negligible for $24 \mathrm{~h}$ (i.e., below the detection limits, $<5 \%$, of XRD and optical microscopy), despite the solution being supersaturated with respect to this form.

Comparing the behavior of systems with SLS and STC showed that crystallization of CBZ(D) occurs earlier and at lower supersaturations in solutions with SLS (Figure 7). In the presence of STC, higher supersaturations were achieved before crystallization of CBZ(D) began to deplete the supersaturation, and the rate of desupersaturation was slower than in the presence of SLS. At

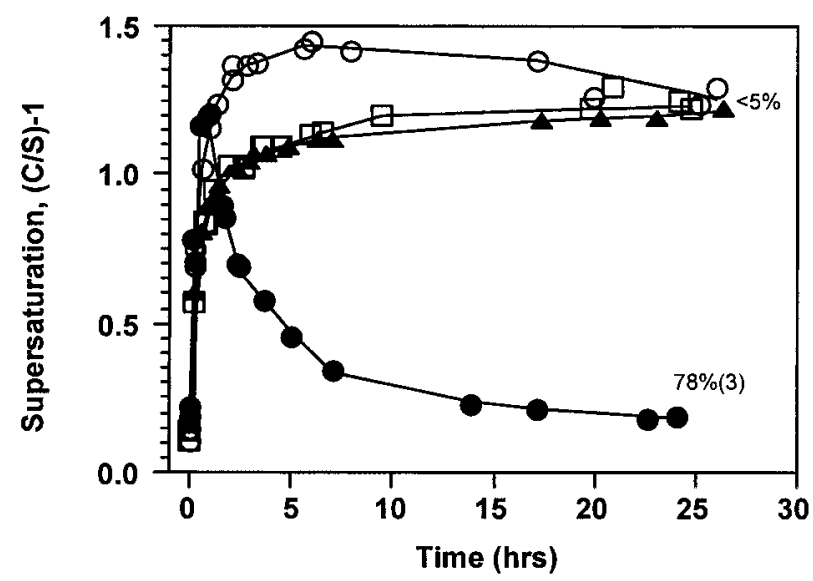

Figure 6. Effect of STC concentration on the supersaturation profile during the transformation of anhydrous to dihydrate $\mathrm{CBZ}$. Initial $(C / S)-1=0.15$ at $25^{\circ} \mathrm{C}$. Key to STC concentration $(\mathrm{mM}):(\mathbf{\Delta}) 0.0 ;(\square) 2.0 ;(\bigcirc)$ 9.0; (O) 17.3. The solid phase composition, \%(w/w) of CBZ(D) in the solid phase with standard deviation in parentheses, is noted for each concentration.

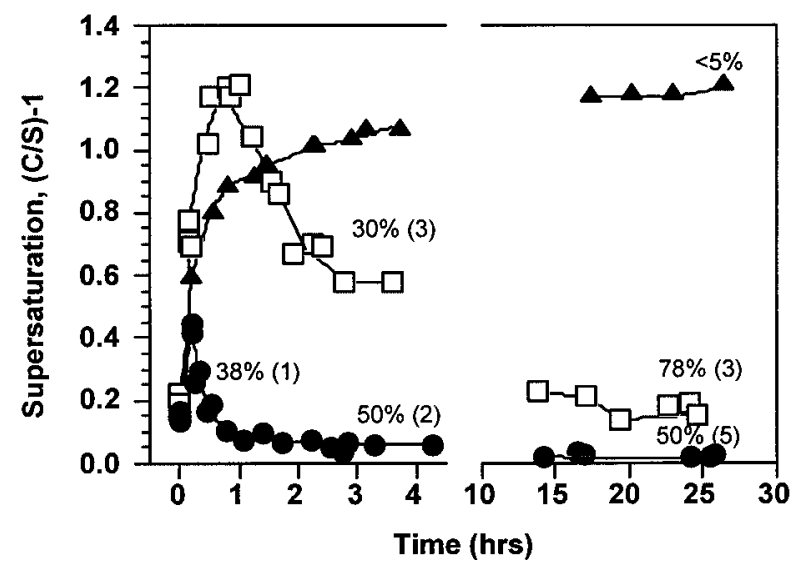

Figure 7. Comparison of surfactant effect on the supersaturation behavior and solid phase composition. Initial $(C / S)-1=0.15$ at $25^{\circ} \mathrm{C}$. Key: ( $\left.\mathbf{\Delta}\right)$ water; $(\square)$ $17.3 \mathrm{mM}$ STC; (O) $17.3 \mathrm{mM}$ SLS. The solid phase composition, \%(w/w) of CBZ(D) in the solid phase with standard deviation in parentheses, is noted for each concentration.

$17.3 \mathrm{mM}$ surfactant concentration, CBZ(D) crystals appeared within $10 \mathrm{~min}$ in SLS compared to $\sim 1 \mathrm{~h}$ in STC. However, a higher fraction of CBZ(A) remained unchanged in solutions with SLS compared with STC; that is, 50 and $22 \%$ CBZ (A) in the solid phase, respectively, and a supersaturation of $\leq 0.2$ within $24 \mathrm{~h}$.

STC significantly changed the crystal morphology of CBZ(D) (Figures 4 and 8), whereas heterogeneous nucleation of CBZ(D) on the surface of the anhydrous form did not occur to a significant extent. XRPD and zero-level precession photographs do not reveal differences in the unit cell dimensions of CBZ(D) grown in solutions with and without STC. There were also no differences in the thermal behavior during dehydration and subsequent melt of CBZ(D) crystallized in the presence or absence of STC. This result suggests that STC was not incorporated within the CBZ(D) crystal lattice and that the crystal morphology change is a result of interactions between STC and specific crystal faces of the dihydrate form.

The characteristic morphology of CBZ(D) crystals grown from water only, SLS, and STC during dissolution of $\operatorname{CBZ}(\mathrm{A})$ is shown in Figure 4. In water only and in SLS solutions, growth along the b-direction, [010], is fastest, and faces that are perpendicular to this direction have very small surface areas. Consequently, CBZ(D) crystals grown from these solutions are needles elongated along the [010] direction. In contrast, CBZ(D) crystals grown during the transformation 


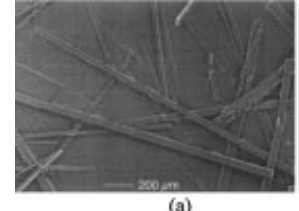

(a)

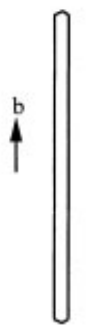

(a)

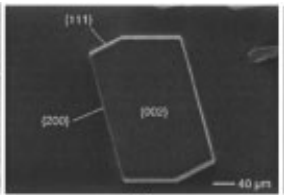

(b)

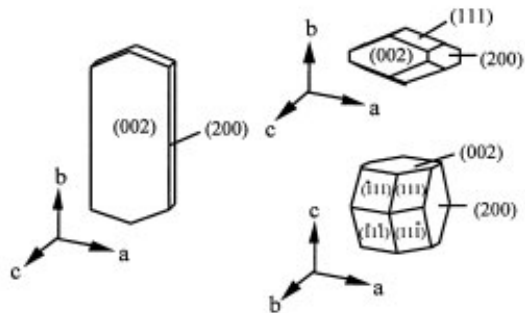

(b)

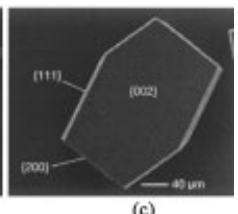

(c)
Figure 8. Crystal morphology of CBZ(D) grown in (a) water, (b) $5 \mathrm{mM} \mathrm{STC}$, and (c) $9 \mathrm{mM}$ STC at constant supersaturation $(C / S)-1=1.5$ and $25^{\circ} \mathrm{C}$.

in $17.3 \mathrm{mM}$ STC show a more isometric shape and diminished growth along the [010] direction.

Single crystal growth experiments, carried out at constant supersaturation of 1.5 during growth, confirmed that CBZ(D) morphology is dependent on STC concentration (Figure 8). Plates were grown in $5.0 \mathrm{mM}$ STC and bipyramids in $9.0 \mathrm{mM}$ STC, with the crystal habit dominated by the $\{111\}$, $\{002\}$, and $\{200\}$ faces. Thus, STC inhibits the relative growth rate of $\mathrm{CBZ}(\mathrm{D})$ along the b-direction, and increases the surface area and morphological significance of $\{111\}$ faces.

\section{DISCUSSION}

In an earlier publication, our group showed that CBZ(A) transforms to CBZ(D) by a solutionmediated process. ${ }^{23}$ In this process, the concentration of drug in solution and the extent of the phase transformation depend on the rate of dissolution of the metastable phase $[\mathrm{CBZ}(\mathrm{A})]$ and the rates of nucleation and growth of the stable phase [CBZ(D)]. We have shown that milling of CBZ(A) changes the rate-controlling process from crystallization of $\mathrm{CBZ}(\mathrm{D})$ to dissolution of $\mathrm{CBZ}(\mathrm{A})$.

In addition to solid-state processing, solutionmediated transformations are sensitive to solution conditions (additives, $\mathrm{pH}$, supersaturation, and temperature). The experimental results presented in this paper indicate that both surfactants, SLS and STC, increase the transformation rate of CBZ(A) to CBZ(D), and that the onset, extent, and mechanism of CBZ(D) crystallization depend on surfactant molecular structure, concentration, and supramolecular association. The general shape of the supersaturation profiles shows that surfactants change the rate-controlling step from crystallization of CBZ(D) to dissolution of CBZ(A) as the surfactant concentration increases and that SLS is more effective than STC in increasing the rate of crystallization. The discussion that follows examines the effect of surfactant structure and concentration on surfactant self-association, interaction with CBZ crystal faces, CBZ solubilization, hydrogen bonding, CBZ(D) crystal morphology, CBZ(D) nucleation, and crystal growth.

Microscopic examination of the solid phases during the transformation revealed that SLS facilitates the surface-mediated nucleation of CBZ(D) on the dissolving CBZ(A). Systems with STC and without surfactants do not exhibit this behavior. The greater extent of heterogeneous nucleation in the presence of SLS may be a result of adsorption of SLS at the CBZ(A) crystal-solution interface and solubilization of CBZ in these adsorbed SLS assemblies. Solubilization of PC at the CBZ(A) crystal-solution interface confirms the presence of surface assemblies of SLS below the bulk cmc capable of adsolubilizing or coadsorbing CBZ, since SLS micelles solubilize CBZ (Figure 2). A number of compounds, including steroids, ${ }^{40}$ styrene, ${ }^{41}$ and dyes, ${ }^{42}$ are solubilized by adsorbed surfactant layers and hemimicelles or two-dimensional micelles on solid surfaces. This solubilization will lead to high interfacial concentrations of CBZ on the dissolving anhydrous surface and provide the driving force for crystallization of CBZ(D) on the surface of CBZ(A). Micelle-mediated crystallization is also supported by the lower supersaturation threshold for nucleation of CBZ(D) at SLS concentrations above the cmc. Luhtala ${ }^{25}$ has reported similar behavior, although transformation studies were done under unstirred conditions by measuring the total particle size distributions of CBZ(A) suspensions. The studies of Luhtala and co-workers show that $\mathrm{SLS}^{25}$ and benzalkonium chloride ${ }^{15}$ accelerate the crystallization of CBZ(D) during dissolution of CBZ(A) at surfactant concentrations above the cmc.

Surfactant assemblies can promote crystallization by acting as templates for the nucleation and growth of crystals ${ }^{14,20}$ and/or by solubilizing, and thus concentrating solute molecules in micelles. ${ }^{17}$ The former mechanism explains the increase in the transformation rates of $\mathrm{CBZ}(\mathrm{A})$ to $\mathrm{CBZ}(\mathrm{D})$ in the presence of STC because there is a small 
increase in the solubilization of CBZ, whereas both templating and solubilization may be effective in the presence of SLS. The latter mechanism was proposed to accelerate the crystallization rate of $\mathrm{CBZ}(\mathrm{D})$ in both benzalkonium chloride and SLS. ${ }^{15,25}$ Nucleation and crystal growth models predict an increase in rates with increasing solubility when the supersaturation is constant., ${ }^{9,4}$ This prediction is due to a decrease in the interfacial tension and an increase in the frequency of molecular collisions with increasing solubility. Surfactants can also retard crystallization when the increase in solubility is compensated by intermolecular interactions between surfactant and crystallizing solute that interfere with the formation and growth of stable nuclei. ${ }^{13,18,19}$

Morphology or crystal shape changes of organic materials have been thoroughly studied ${ }^{31,44,45}$ and are due to different crystal faces that can grow at different rates depending on the solvent, additives, impurities, and supersaturation. When growth of a crystal face is inhibited, the surface area of this face is expected to increase relative to the areas of other faces, because growth of a face is defined as the rate at which the face grows perpendicular to itself. In this way, crystal morphology changes, and the well-defined arrangement of molecules on crystal surfaces offer a means to probe molecular recognition events at interfaces. CBZ(D) grows as needles with fastest growth along the b-axis in the absence of surfactant and in systems with SLS, whereas it grows as prismatic crystals with welldefined $\{111\},\{002\}$, and $\{200\}$ faces and decreased growth rate along the b-axis in the presence of STC (Figures 4 and 8). This change in morphology suggests specific interactions between STC and the functional groups on the $\{111\}$ faces of CBZ(D).

To understand this preferential interaction, we examined the crystal structure of CBZ(D), the molecular interactions along morphologically important faces, and the conformation and supramolecular structures of STC. The crystal structure of $\mathrm{CBZ}(\mathrm{D})$ is characterized by hydrogen bonds forming dimers, chains, and tapes. ${ }^{32,46}$ Significant interactions of the $\{111\}$ faces and along the b-direction of CBZ(D) crystals include a channel of hydrogen-bonded water molecules and $\pi-\pi$ interactions between the aromatic regions of CBZ molecules (Figure 9). This direction of growth is expected to be the fastest, in the absence of solvent or additive effects, because it has the strongest intermolecular interactions and the shortest unit cell dimensions. CBZ molecules form centrosymmetric dimers by hydrogen bonding between

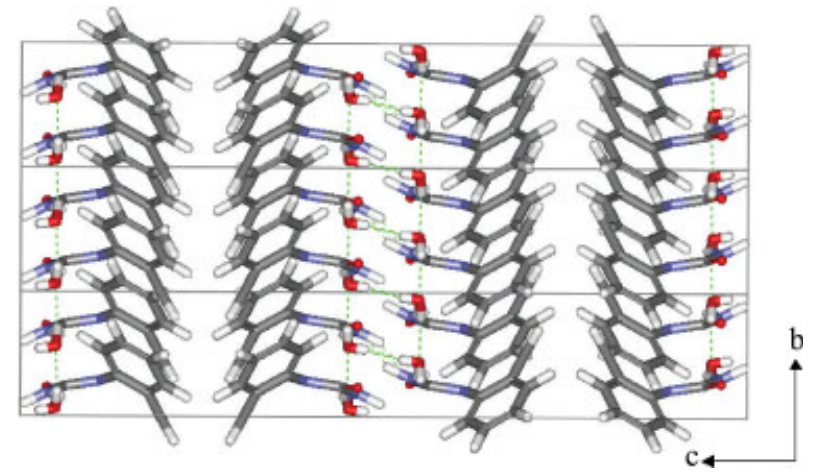

Figure 9. $\mathrm{CBZ}(\mathrm{D})$ crystal lattice viewed down the a-axis, showing the hydrogen bonding along the b-axis. Space group Cmca, unit cell dimensions: $a=19.748 \AA$, $\mathrm{b}=4.924 \AA, \mathrm{c}=28.659 \AA, \alpha=\beta=\gamma=90^{\circ}$.

carboxamide groups along the c-axis (Figure 10). Growth of faces perpendicular to this axis, $\{002\}$ faces, involves hydrogen-bonded homodimers between CBZ molecules and water molecules. Growth perpendicular to the $\{200\}$ faces and along the a-direction results from hydrogen bonding between water molecules and carboxamide groups forming a tape. Morphology predictions will be addressed in a subsequent publication. Based on unit cell dimensions and attachment energy calculations, morphology predictions identified the $\{111\},\{002\}$, and $\{200\}$ faces as morphologically significant and the $\mathbf{b}$-axis as the longest crystal dimension, which is in agreement with the experimental results presented here. ${ }^{47,48}$

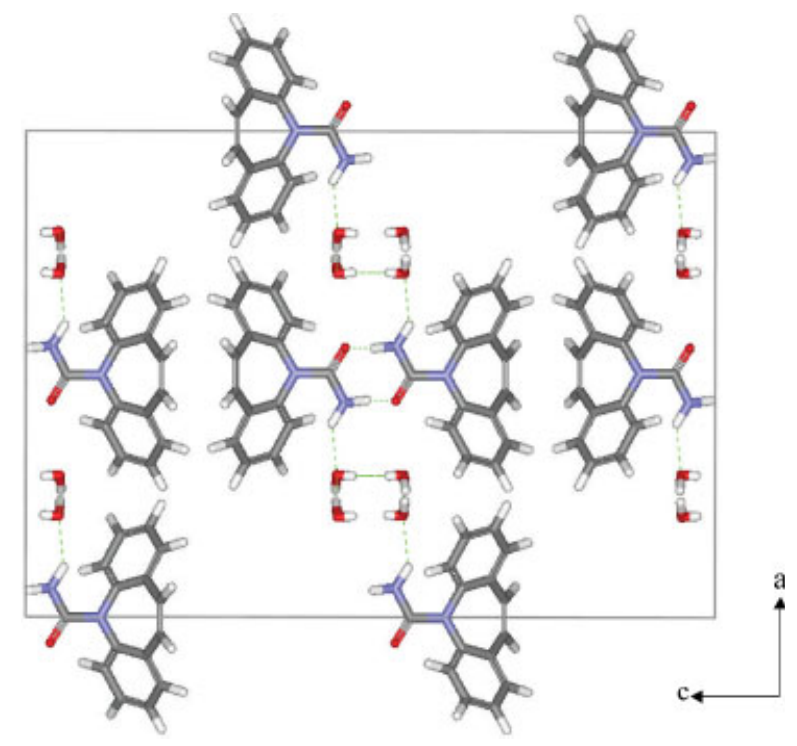

Figure 10. CBZ(D) crystal lattice viewed down the b-axis. A molecular tape is formed between alternating CBZ dimers and water molecules. 
Examination of bile acid supramolecular assemblies in aqueous solutions and in the crystalline state conveys valuable information about the relationship between molecular structures and resultant micelles or supramolecular structures. STC molecules exhibit facial polarity, with the hydrophilic part consisting of three hydroxyl groups and a sulfonate group situated below the equator of the rigid steroid ring, and with the hydrophobic methyl groups situated above this equator ${ }^{28,49}$ (Figures 1 and 11). In contrast to alkyl chain surfactants, such as SLS, that exhibit axial polarity, well-defined cmc values, and homogeneous distribution of micellar aggregates with aggregation numbers of at least $50,{ }^{50}$ bile salts exhibit a wide range of $\mathrm{cmc}$ values because of their progressive association and heterogeneous distribution of aggregates. ${ }^{29,38,51}$ In the case of STC, micellar aggregates are dimers or tetramers, with the hydrophobic surfaces of the molecules back-toback towards the interior of the aggregate and the hydrophilic groups in contact with the aqueous environment. ${ }^{27,28}$ At concentrations STC is $<50 \mathrm{mM}$, distributed as monomers, dimers, and perhaps tetramers. ${ }^{28}$

In addition to hydrophobic interactions, intermolecular hydrogen bonding has also been proposed to occur in larger bile salt oligomers, ${ }^{28,29}$ by considering nonpolar and polar interactions that stabilize these assemblies in both liquid and crystalline states. STC hydrate crystals have bilayered structures with hydrophilic and hydrophobic regions formed through intralayered, cyclic hydro-

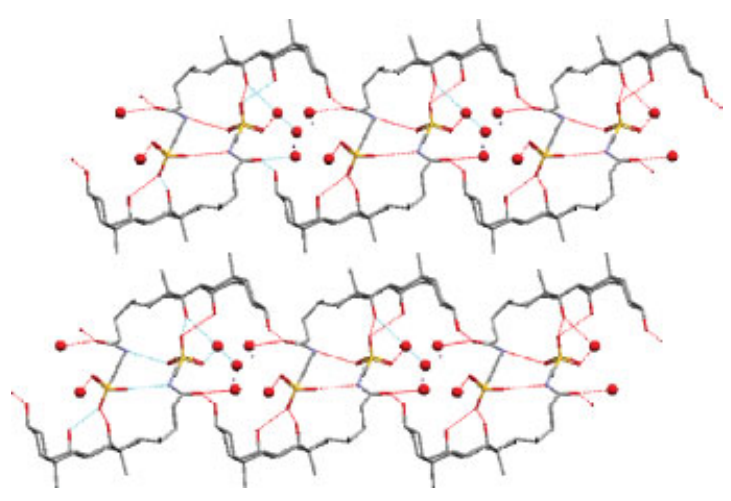

Figure 11. Bilayers in hydrated STC crystal comprise hydrophobic and hydrophilic layers with extensive hydrogen bonds involving hydroxyl and taurine groups of STC molecules and water molecules. Water oxygens are shown as large spheres and sodium ions as small spheres. Hydrogen atoms are omitted for the sake of clarity. gen bonds ${ }^{49,50}$ (Figure 11). The hydrophilic intralayer constitutes extensive hydrogen bonding with hydroxyl groups of the steroid moiety, the amino and sulfonate groups of the taurine chain, and water, whereas the hydrophobic layer is composed of the steroid structure and the methyl groups. Stacking of the hydrophobic sides of STC in the crystal is analogous to their assembly in aqueous solutions. Considering the structure of STC assemblies at the bile salt concentrations studied here and the molecular arrangement of CBZ on morphologically important faces, the morphology change in the presence of STC can be explained by hydrogen bonding between the hydroxyl and sulfonate groups of STC and the lattice water and carboxamide group exposed on the surface of the $\{111\}$ faces of CBZ(D) crystals. The dependence of morphology change on STC concentration and the structure of surfactant assemblies in solution with exposed functional groups that can form hydrogen bonds under these conditions support the proposed model.

\section{CONCLUSIONS}

The results of this study show that surfactants affect the solution-mediated transformation of drugs in significant ways, and that the rate and mechanism of the conversion depends on the molecular and supramolecular structures of surfactants in solution and at crystal-solution interfaces. Both surfactants, SLS and STC, increased the anhydrous-to-dihydrate CBZ transformation in aqueous solutions depending on the type and concentration of surfactant. SLS promoted the surface-mediated nucleation of CBZ(D) on the CBZ(A) crystals at surfactant concentrations below the cmc and had little effect of CBZ(D) morphology. Above the cmc, both surfactants promoted the transformation by increasing the bulk nucleation of CBZ(D). STC changed the crystal morphology of CBZ(D) from acicular to prismatic, depending on STC concentration. Such morphology changes originate from interactions between STC and molecular structures of CBZ(D) crystal faces that interfere with the formation of a hydrogen-bonded chain of water molecules and carboxamide dimers. The hydrogen bonding potential of the surfactant has a significant effect on the morphology of CBZ(D) crystals. Compared with SLS, with only hydrogen bond acceptor potential, STC has four hydrogen bond donor groups and three hydrogen bond acceptors. The 
results of this study emphasize the important role of surfactants and crystallization in determining the dissolution behavior of metastable solid forms of a drug substance and in developing meaningful dissolution methods.

\section{ACKNOWLEDGMENTS}

This material is based on work supported by National Science Foundation Grant 9526328 and the Vahlteich Research Award from the College of Pharmacy, University of Michigan. The scanning electron microscope used in this study was funded in part by National Science Foundation Grant EAR-9628196. Financial support from the Horace Rackham School of Graduate Studies, University of Michigan, and the Fred W. Lyons Jr. Fellowship from the College of Pharmacy, University of Michigan is appreciated.

\section{REFERENCES}

1. Byrn S, Pfeiffer R, Ganey M, Hoiberg C, Poochikian G. 1995. Pharmaceutical solids-A strategic approach to regulatory considerations. Pharm Res 12: 945-954.

2. Shekunov BY, York P. 2000. Crystallization processes in pharmaceutical technology and drug delivery design. J Cryst Growth 211:122-136.

3. Rodríguez-Hornedo N, Lechuga-Ballesteros D, Wu HJ. 1992. Phase-transition and heterogeneous epitaxial nucleation of hydrated and anhydrous theophylline crystals. Int J Pharm 85:149-162.

4. Kobayashi Y, Ito S, Itai S, Yamamoto K. 2000. Physicochemical properties and bioavailability of carbamazepine polymorphs and dihydrate. Int J Pharm 193:137-146.

5. Vippagunta SR, Brittain HG, Grant DJW. 2001. Crystalline solids. Adv Drug Delivery Rev 48:3-26.

6. Byrn SR, Xu W, Newman AW. 2001. Chemical reactivity in solid-state pharmaceuticals: formulation implications. Adv Drug Delivery Rev 248:115-136.

7. Shalev E, Shaleva M, Zografi G. 2002. The effect of disorder on the chemical reactivity of an organic solid, tetraglycine methyl ester: Change of the reaction mechanism. J Pharm Sci 91:584-593.

8. Davey RJ, Cardew PT, McEwan D, Sadler DE. 1986. Rate controlling processes in solventmediated phase-transformations. J Cryst Growth 79:648-653.

9. Rodríguez-Hornedo N, Murphy D. 1999. Significance of controlling crystallization mechanisms and kinetics in pharmaceutical systems. J Pharm Sci 88:651-660.
10. Crison JR, Shah VP, Skelly JP, Amidon GL. 1996. Drug dissolution into micellar solutions: Development of a convective diffusion model and comparison to the film equilibrium model with application to surfactant-facilitated dissolution of carbamazepine. J Pharm Sci 85:1005-1011.

11. Crison JR, Weiner ND, Amidon GL. 1997. Dissolution media for in vitro testing of water-insoluble drugs: Effect of surfactant purity and electrolyte on in vitro dissolution of carbamazepine in aqueous solutions of sodium lauryl sulfate. J Pharm Sci 86: 384-388.

12. Luner PE. 2000. Wetting properties of bile salt solutions and dissolution media. J Pharm Sci 89: 382-395.

13. Dugua J, Simon B. 1978. Crystallization of sodium perborate from aqueous solutions. II. Growth kinetics of different faces in pure solution and in the presence of surfactant. J Crystal Growth 44:280286.

14. Weissbuch I, Addadi L, Leiserowitz L, Lahav M. 1988. Total asymmetric transformations at interfaces with centrosymmetric crystals: Role of hydrophobic and kinetic effects in the crystallization of the system glycine/ $\alpha$-amino acids. J Am Chem Soc 110:561-567.

15. Luhtala S, Kahela P, Kristofferson E. 1990. Effect of benzalkonium chloride on crystal growth and aqueous solubility of carbamazepine. Acta Pharm Fennica 99:59-68.

16. Luhtala S. 1992. Effect of poloxamer 184 on crystal growth and aqueous solubility of carbamazepine. Acta Pharm Nord 4:271-276.

17. Reddy KSN, Salvati LM, Dutta PK, Abel PB, Suh KI, Ansari RR. 1996. Reverse micelle based growth of zincophosphate sodalite: Examination of crystal growth. J Phys Chem 100:9870-9880.

18. Garti N, Zour H. 1997. The effect of surfactants on the crystallization and polymorphic transformation of glutamic acid. J Crystal Growth 172:486-498.

19. Füredi-Milhofer H, Tunik L, Filipovic-Vincekovic N, Skrtic D, Babic-Ivancic V, Garti N. 1995. Induction of crystallization of calcium oxalate dihydrate in micellar solutions of anionic surfactants. Scanning Microscopy 9:1061-1070.

20. Bujan M, Sikiric M, Filipovic-Vincekovic N, Vdovic N, Garti N, Füredi-Milhofer H. 2001. Effect of anionic surfactants on crystal growth of calcium hydrogen phosphate dihydrate. Langmuir 17:6461-6470.

21. Kaneniwa N, Ichikawa JI, Yamaguchi T, Hayashi K, Watari N, Sumi M. 1987. Dissolution behavior of carbamazepine polymorphs. Yakugaku Zasshi (Journal of the Pharmaceutical Society of Japan) 107:808-813.

22. Young WWL, Suryanarayanan R. 1991. Kinetics of transition of anhydrous carbamazepine to carbamazepine dihydrate in aqueous suspensions. J Pharm Sci 80:496-500. 
23. Murphy D, Rodríguez-Cintrón F, Langevin B, Kelly RC, Rodríguez-Hornedo N. 2002. Solutionmediated phase transformation of anhydrous to dihydrate carbamazepine and the effect of lattice disorder. Int J Pharm 246:121-134.

24. USP. 1995. The United States Pharmacopeia (USP 23). Rockville, MD: United States Pharmacopieal Convention, Inc.

25. Luhtala S. 1992. Effect of sodium lauryl sulphate and polysorbate 80 on crystal growth and aqueous solubility of carbamazepine. Acta Pharm Nord 4: 85-90.

26. Luhtala S, Kinos E, Yliruusi J, Tanninen VP. 1992. X-ray powder diffraction study of carbamazepine crystals grown in surfactant solutions. Acta Pharm Nord 4:283-290.

27. Coello A, Meijide F, Nuñez ER, Tato JV. 1996. Aggregation behavior of bile-salts in aqueoussolution. J Pharm Sci 85:9-15.

28. Bottari E, D'Archivio AA, Festa MR, Galantini L, Giglio E. 1999. Structure and composition of sodium taurocholate micellar aggregates. Langmuir 15:2996-2998.

29. Bonincontro A, D'Archivio AA, Galantini L, Giglio E, Punzo F. 2000. X-ray, electrolytic conductance, and dielectric studies of bile salt micellar aggregates. Langmuir 16:10436-10443.

30. Li L, Rodríguez-Hornedo N. 1992. Growth kinetics and mechanism of glycine crystals. J Crystal Growth 121:33-38.

31. Lechuga-Ballesteros D, Rodríguez-Hornedo $\mathrm{N}$. 1995. Effects of molecular structure and growth kinetics on the morphology of -alanine crystals. Int J Pharm 115:151-160.

32. Reck G, Dietz G. 1986. The order-disorder structure of carbamazepine dihydrate- 5 H-dibenz [B, F]azepine-5-carboxamide dihydrate. Cryst Res Technol 21:1463-1468.

33. Nunn CC, Schechter RS, Wade WH. 1982. Visual evidence regarding the nature of hemimicelles through surface solubilization of pinacyanol chloride. J Phys Chem 86:3271-3272.

34. Mukerjee P, Mysels KJ. 1955. A re-evaluation of the spectral change method of determining critical micelle concentration. J Am Chem Soc 77:29372943.

35. Sato H, Kawasaki K. 1983. Energy-transfer between rohodamine- $6 \mathrm{~g}$ and pinacyanol enhanced with sodium dodecyl sulfate in the premicellar region-formation of dye-rich induced micelles. J Phys Chem 87:3759-3769.

36. Adamson AW. 1990. Physical chemistry of surfaces. New York: John Wiley \& Sons, pp 4-52.

37. Mukerjee P. 1967. The nature of the association equilibria and hydrophobic bonding in aqueous solutions of associated colloids. Adv Colloid Interface Sci 1:241-250.
38. Carey MC, Small DM. 1972. Micelle formation by bile salts. Arch Intern Med 130:506-527.

39. Yalkowsky SH. 1999. Solubility and solubilization in aqueous media. New York: Oxford University Press, pp 262-266.

40. Jansen J, Treiner C, Vaution C. 1996. Coadsorption of steroids and nonionic surfactants on polystyrene latex particles from aqueous solutions. J Colloid Interface Sci 179:578-586.

41. Wu J, Harwell JH, O'Rear EA. 1987. Two-dimensional solvents: Kinetics of styrene polymerization in admicelles at or near saturation. J Phys Chem 91:623-634.

42. Mukerjee P, Sharma R, Pyter RA, Gumkowski MJ. 1995. Adsorption of surfactants and solubilization in adsorbed layers. ACS Symp Ser 615:22-35.

43. Gu CH, Young V, Grant DJW. 2001. Polymorph screening: Influence of solvents on the rate of solvent-mediated polymorphic transformation. J Pharm Sci 90:1878-1890.

44. Zipp GL, Rodríguez-Hornedo N. 1993. Growth mechanism and morphology of phenytoin and their relationship with crystallographic structure. J Phys D: Appl Phys 26:B48-B55.

45. Weissbuch I, Lahav M, Leiserowitz L. 1999. Towards an understanding and control of nucleation, growth, habit, dissolution, and structure of crystals using "tailor-made" auxiliaries. In Myerson AS, editor. Molecular modeling applications in crystallization. New York: Cambridge University Press, pp 166-227.

46. Morris KR, Griesser UJ, Fanwick P, personal communication.

47. Murphy D. 1997. The solvent-mediated phase transformation of carbamazepine and the influence of surfactants on the nucleation mechanism and crystal growth. Ph.D. Thesis. University of Michigan, Ann Arbor, MI.

48. Sinclair BD. 2000. Interpreting changes in nucleation and crystal morphology of carbamazepine dihydrate by probing the intermolecular interactions between additives and crystal surfaces. Ph.D. Thesis. University of Michigan, Ann Arbor, MI.

49. Campanelli AR, Desanctis SC, D'Archivio AA, Giglio E, Scaramuzza L. 1991. Crystal-structures of bile-salts-sodium taurocholate. J Inclusion Phenom 11:247-256.

50. Kratohvil JP. 1980. Comments on some novel approaches for the determination of micellar aggregation numbers. J Colloid Interface Sci 75:271-275.

51. Li Y, Holzwarth JF, Bohne C. 2000. Aggregation dynamics of sodium taurocholate and sodium deoxycholate. Langmuir 16:2038-2041.

52. Briganti G, D’Archivio AA, Galantini L, Giglio E. 1996. Structural study of the micellar aggregates of sodium and rubidium glyco- and taurodeoxycholate. Langmuir 12:1180-1187. 